Это еще один плюс использования сетевой технологии для решения задач, поставленных в этой работе. Таким образом хорошим решением для многих из перечисленных проблем являются:

1. Использование интернет-технологии и создание web-приложения;

2. Поэтапное преподнесения информации и поэтапный контроль знаний;

3. Глазной итоговый контроль знаний;

4. Хранение вопросов и ответов, проб и результатов тестирования в базе данных, расположенной на wеbсервере, доступ к просмотру и редактированию которой есть только у определенного круга лиц;

5. Разграничение администраторской и клиентской частей приложения;

6. Использование для разработки системы скриптовых языков программирования, формируют страницу с данными непосредственно на web-сервере. Пользователь системы получает страницу, содержащую текст подготовлен лично для него.

Научный руководитель: стариий преподаватель Дец Д.В.

\title{
Литература
}

1. Адлер Ю.П., Кочетов А.И. Повышение качества подготовки специалистов.// Стандарты и качество, МИСиС - 2000.- № 2.- С. 68-72;

2. Куклев, В. А. Методология мобильного обучения. - Ульяновск: УлГТУ, 2006. - 254 с.

\section{ИСПОЛЬЗОВАНИЕ ШАБЛОНА ПРОЕКТИРОВАНИЯ MVC ДЛЯ СОЗДАНИЯ ВЕБ-ПРИЛОЖЕНИЙ}

\author{
Щербакан Д.В. ${ }^{1}$ \\ ${ }^{1}$ Одеська Національна академія харчових технологій, Одеса.
}

Copyright (C) 2014 by author and the journal “Automation technological and business - processes". This work is licensed under the Creative Commons Attribution International License (CC BY). http://creativecommons.org/licenses/by/4.0/

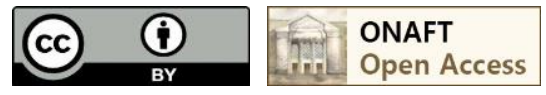

\section{Аннотация \\ Ррассматриваются общие принципы построения приложений с использованием шаблона проектирования MVC. Указываются некоторые особенности его использования в веб-разработке.}

\begin{abstract}
The general principles for building applications using the MVC design pattern are reviewing. Some features of using it in web-development are specifying.
\end{abstract}

Ключевые слова

Программирование, MVC, веб-разработка.

Постановка проблемы.

На данном этапе развития интернета, все чаще веб-приложения должны реализовывать сложную бизнеслогику, передавать и обрабатывать логически связанные данные, автоматически реагировать на возникновение ошибок. При этом необходимо сохранить возможность быстрого изменения или добавления функционала 


\section{7 СТУДЕНТСЬКА НАУКА}

приложения, будь то какой-то элемент внутренней логики, обработка обращения в серверу или отображение информации в браузере пользователя. Для решение этой задачи разработчики широко используют шаблон проектирования MVC.

Формирование цели статьи.

Дать общее представление о шаблоне MVC, указать об особенностях его применения для разработки вебприложений.

\section{Основной материал.}

Шаблон проектирования MVC предполагает разделение данных приложения, пользовательского интерфейса и управляющей логики на три отдельных компонента (модель, представление, контроллер) таким образом, что модификация каждого компонента не повлияет на другие его компоненты. Модель (Model) предоставляет данные предметной области представлению и реагирует на команды контроллера, изменяя свое состояние. Представление (View) отвечает за отображение данных предметной области (модели) пользователю, реагируя на изменения модели. Контроллер (Controller) интерпретирует действия пользователя, оповещая модель о необходимости изменений[1]. То есть это способ создания веб-сайтов/приложений с четким разделением кода - размещение бизнес-логики и доступа к данным в одном месте, код представления (например, HTML-разметку) в другом, и связующее звено между ними в третьем. MVC также идет рука об руку с объектно-ориентированным программированием. Для некоторых проектов, MVC и ООП является излишеством - сайт-визитку с одной контактной формой можно сделать гораздо проще. Тем не менее, для любого более-менее серьезного проекта в котором необходим доступ к базе данных, использование пользовательских сессий, сторонних библиотек, обработка больших объемов информации, шаблон MVC является хорошим выбором.

Для любого веб-приложения, написанного, с использованием шаблона MVC, можно выделить несколько общих особенностей:

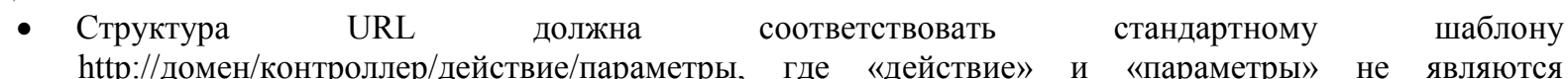
обязательными.

- Страница «index.php» - наша главная «целевая страница», которая является единой точкой входа для всех запросов.

- $\quad$ index.php создаст «объект-загрузчик», который отвечает за распознавание запрашиваемого в URL контроллера, а затем выполнение запрошенного действия (т.е. метода) этого контроллера.

- Действия контроллера отвечают за создание экземпляра соответствующей модели и отправки данных из модели в соответствующее представление.

- Все контроллеры, модели и представления - это отдельные файлы, организованные в папки с соответствующими именами. Папка представлений имеет файл, соответствующий каждому контроллеру. В файлах контроллеров и моделей находятся соответствующие им классы или отдельные функции, в то время как файлы просмотров файлы, в основном, содержат разметку (XML, HTML и т.д.).

- У контроллеров и моделей есть «базовый класс», который они наследуют (расширяют).

Ключевые элементы такого приложения и их взаимосвязь продемонстрированы на рисунке 1. 


\section{ㄱ СТУДЕНТСЬКА НАУКА}

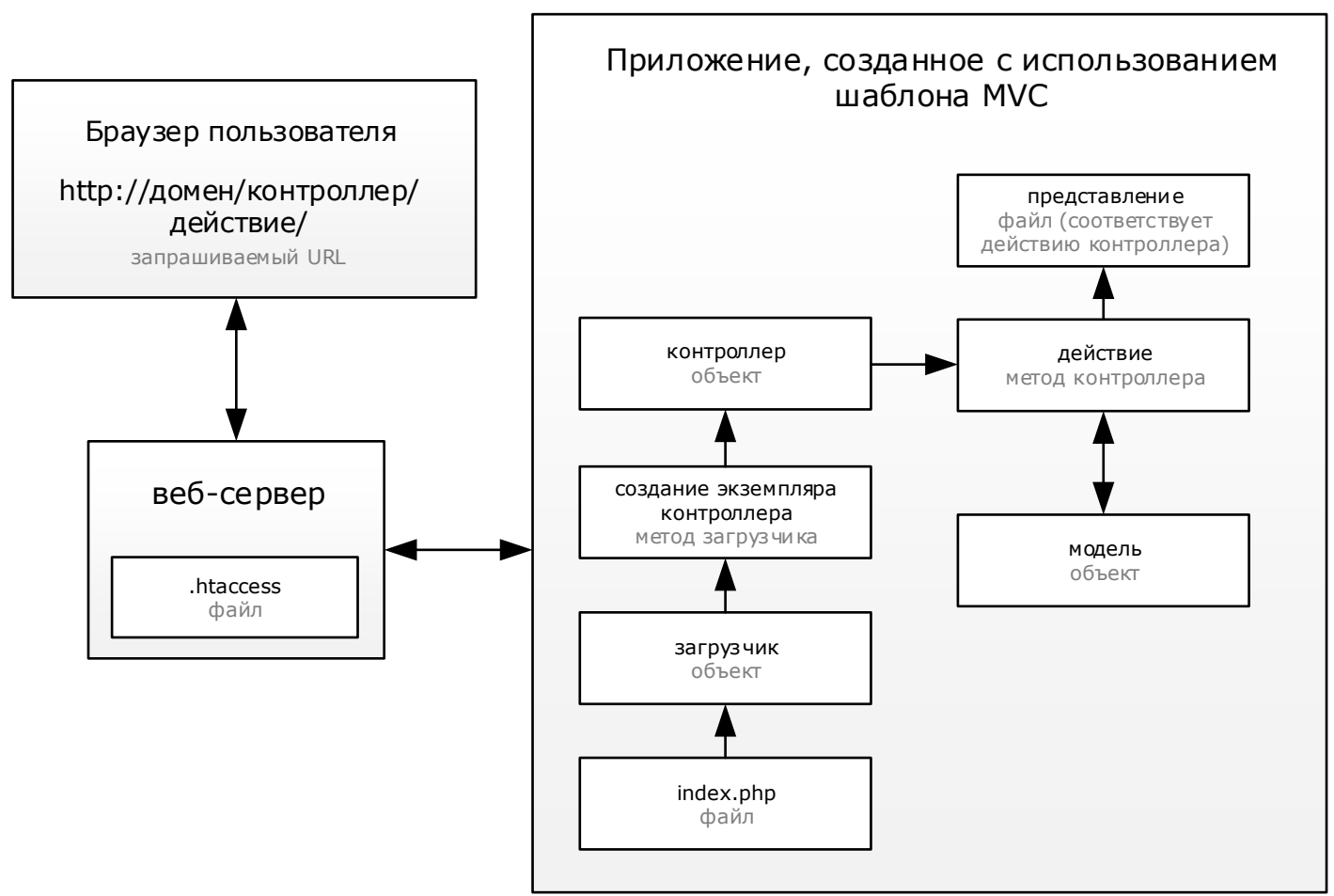

Рис.1. Ключевые элементы приложения, реализованного с использованием шаблона MVC и их взаимосвязь.

Одной из общих особенностей приложений, реализованных таким образом, является структура URLадресов - http:/домен/контроллер/действие. Для ее реализации, должна использоваться перезапись URL. K примеру, в веб-сервере «Арасhе», перезапись URL доступна благодаря модулю «mod_rewrite» [2].

Основной задачей данного модуля, в контексте рассматриваемого вопроса, является перенаправление всех запросов пользователя к файлу «index.php». В этом файле создается объект-загрузчик, который проводит анализ полученного запроса, создает необходимый экземпляр контроллера и вызывает его действие. В случае, если запрошенный контроллер не найден, то в объекте-загрузчике происходит обработка ошибки. Если в выбранном контроллере не найдено необходимое действие, то в нем обрабатывается ошибка. Для обработки ошибок приложения могут использовать отдельные объекты, которые предоставляют другим разработчикам или конечному пользователю более подробную информацию о месте и причине и возникновения ошибки.

В приложениях, написанных с использованием шаблона MVC, контроллер является связующим звеном между моделями и представлениями. Он «говорит» модели, какие действия над данными необходимо произвести, и он передает ее ответ представлению, которое занимается только отображением этих данных конечному пользователю. Так как разные контроллеры могут выполнять одинаковые функции, то имеет смысл создавать один «базовый» контроллер, в котором они и будут определены. Все остальные контроллеры просто будут наследовать базовый. Такое решение позволяет избежать копирования одинаковых участков программного кода и предоставляет больше удобства для добавления новых функций, или изменения уже имеющихся.

В реальных приложениях постоянно появляется необходимость обработки записей из базы данных, заголовков страниц, HTML метаданных, данных сессии и т.д. Если реализацию этого функционала размещать в контроллерах, то очень скоро они разрастутся до таких размеров, что поддержка кода станет чрезвычайно сложным занятием. Чтобы этого не происходило в приложениях и используются модели.

В контексте шаблона MVC, модель - это объект, который создается в методе контроллера для предоставления доступа к данным, их изменения или удаления. Количество моделей и их взаимосвязь с контроллерами зависит от каждой конкретной задачи. В одних проектах может быть целесообразно создать одну модель для всех контроллеров. В других - каждая модель может соответствовать таблице базы данных.

Модели, как и контроллеры, стоит наследовать от «базового» класса моделей. Это делается по тем же причинам, по которым используется наследование для контроллеров. 
Последним элементом шаблона MVC является представление, которое ответственно за отображение на экране данных полученных из модели. Обычно для этого используются шаблоны целых HTML-страниц или отдельных их элементов. При этом никакая обработка данных в представлении не должна проводиться. Программный код в представлении должен быть использован только для организации циклов, проверки условий или вывода на экран переменных.

По количеству отображаемой информации представления можно разделить так:

- Общий шаблон. Файл или их совокупность, в которых находится общее содержимое для каждой страницы (шапка сайта, меню, подвал);

- Часть шаблона. Некоторая совокупность функциональных элементов сайта, которые вместе решают определенные задачи. Примером может быть;

- Помощники. Классы, которые можно использовать для отображения отдельных элементов интерфейса, таких как кнопки, ссылки, поля ввода.

Выводы.

Шаблон MVC является хорошей архитектурной основой для сложного веб-приложения, фреймворка или CMS. Его грамотное использование позволит создать продукт, который будет легко изменять и расширять, затратив на это меньше времени и усилий.

Научный руководитель: профессор Хобин Виктор Андреевич

Литература

1. Обобщенный Model-View-Contoller. http://www.rsdn.ru/article/patterns/generic-mvc.xml;

2. Apache Module mod_rewrite http://httpd.apache.org/docs/current/mod/mod_rewrite.html.

\title{
РАЗРАБОТКА МАТЕМАТИЧЕСКОЙ МОДЕЛИ САУ УРОВНЯ МЕТАЛЛА В КРИСТАЛЛИЗАТОРЕ МНЛ3
}

\section{Гарматенко И.А. ${ }^{1}$}

${ }^{1}$ ГВУЗ «Донецкий национальный технический университет», г. Донецк

Copyright (C 2014 by author and the journal “Automation technological and business - processes". This work is licensed under the Creative Commons Attribution International License (CC BY). http://creativecommons.org/licenses/by/4.0/

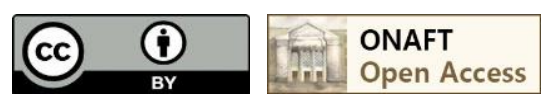

\begin{abstract}
Аннотация
Рассматривается система регулирования уровнем металла в кристаллизаторе при наличии люфта в стопорном механизме. Предложено два способа компенсации люфта. Методом компьютерного моделирования установлено, что применение комбинированной системы регулирования позволяет значительно уменышить отклонение уровня от требуемого значения в переходных режимах работы.
\end{abstract}

\section{Abstract}

Consider the system of of regulation metal level in the mold in the presence of backlash in the locking mechanism. Suggested two ways backlash. Method of computer modeling found that the use of the combined 